\section{§61. Behavior of Helium Gas in the LHD Vacuum Chamber}

Suzuki, H., Ohyabu, N., Komori, A., Miyazawa, J., Sakamoto, R., Kubota, Y., Motojima, O.

\section{Introduction}

It is well known that helium atoms cannot exist in a metal wall like a hydrogen atoms. And helium gas is easily released from a surface of a wall, because of its small desorption activation energy of $590 \mathrm{Jmol}^{-1}$. So it had been believed that helium gas is not trapped in a vacuum vessel so much. However, in a case of a plasma vacuum vessel, a behavior of helium atoms is not the same. Implantations of high energy helium atoms have been reported.

In the LHD plasma vacuum chamber, desorption and trapping of helium atoms has been also observed. Main plasma discharge experiments of the LHD have been carried out using hydrogen and helium gas. The plasma has been produced in every 3 minutes. The discharge time of each shots has been a few second in a most case. After the main plasma discharge experiments, $\mathrm{He}$ GDC or $\mathrm{H}_{2}$ GDC has been carried out at night. The GDC has been stopped at 3:00 a.m., and then the vacuum vessel has been pumped out. However after 7 hour pumping, the main gas in the vacuum vessel has been still helium gas after a He GDC. During the helium gas plasma discharge experiments, about a half of inlet helium gas has been disappear. And during the hydrogen plasma experiment, a helium gas desorption has been observed by not only a mass spectrometer but also an optical spectrometer.

\section{Outgassing of helium after He GDC}

Fig1 shows partial pressures of the helium gas at the starting time of the main plasma discharge experiment, which has been about 7 hours later from GDC ending time. It is clear that the partial pressure of the helium gas has been small after a $\mathrm{H}_{2}$ GDC. After a $\mathrm{He}$ GDC, the helium pressure has been almost same level at every morning. No dependence has been found between the helium pressure and the last main plasma experiment. The helium pressure at the morning has been determined only by the last GDC. The outgassing rate has been estimated to be $1.7 \times 10^{-4} \mathrm{Pam}^{3} / \mathrm{s}$. Assuming the surface area of helium trapping is $730 \mathrm{~m}^{2}$, the outgasing rate per unit area is $2.3 \times 10^{-11} \mathrm{Pam}^{3} / \mathrm{s} / \mathrm{cm} 2$

\section{Implantation of the helium atoms during helium plasma experiments}

Before the main plasma discharge experiment, a test of gas puff valve has been carried out without plasma heating. In this time, no missing helium has been observed. It points out that high energy helium atom is a key of missing atoms. So the missing has been caused by implantation, not a simple surface absorption. At the first campaign of the LHD experiment, no missing of the helium gas had been reported. However the missing of a helium gas has been observed, when plasma stored energy has been increased. Although the wall had been saturated by helium atoms after He GDC, still the missing of the helium atoms has been observed.

Assuming of no wall pumping, a pressure after a plasma discharge can be estimated by total amount of inlet gas and of pumped out gas. Fig2 shows ratios of the measured gas pressure and the calculated gas pressure with no wall pumping assumption of each plasma shots as a function of the plasma stored energy. If the wall does not pump a helium gas, this ratio is one. In a most of case, the ratios are about 0.5 . So almost half of inlet helium gas has been implanted into the wall. It was also found by analyzing a particle balance in a whole plasma experiment, that the implanted helium had been stayed in the wall until the end of the plasma experiment.

\section{Summary}

In the LHD vacuum chamber, helium atoms has been implanted during plasma discharge experiments and $\mathrm{He}$ GDC. The helium gas desorption rates in the morning are almost same level. No dependence between the desorption level and the last plasma experiments has been found. This means that the helium atoms implanted during the plasma experiments had been released during the GDC, and the wall condition has been recovered.

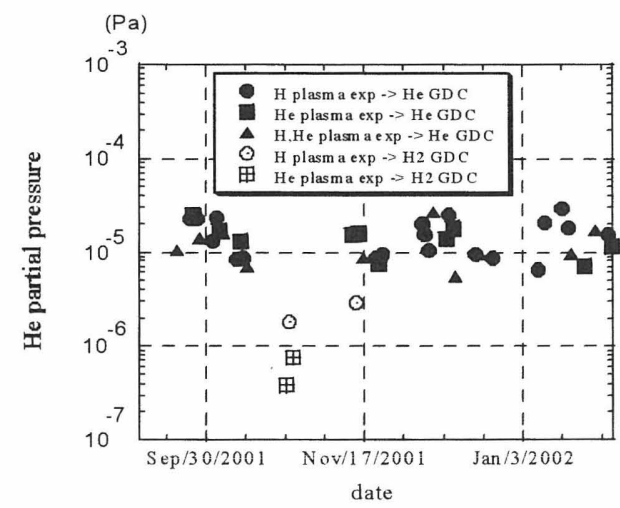

Fig. 1 Helium partial pressure at the starting time of plasma experiment

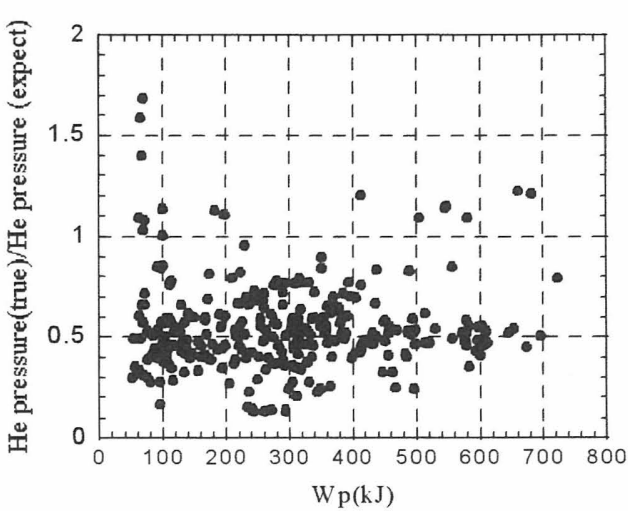

Fig.2 Ratio of measured helium gas pressureand expected pressure with no wall pump assumntion 